\title{
Current Applications of Mesenchymal Stem Cells for Cartilage Tissue Engineering
}

\author{
Lizeth Fuentes-Mera, Alberto Camacho, \\ Nidia K. Moncada-Saucedo and \\ Víctor Peña-Martínez \\ Additional information is available at the end of the chapter \\ http://dx.doi.org/ 10.5772/intechopen.68172
}

\begin{abstract}
Articular cartilage injuries caused by traumatic/mechanical progressive degeneration result in joint pain, swelling, the consequent loss of joint function, and eventually osteoarthritis. Articular tissue possesses a poor ability to regenerate that further complicates the therapeutic approaches. Mesenchymal stem cells (MSCs) have emerged as a promising alternative treatment. Recently, it has been reported that a wide variety of strategies ranging from merely using cells in the injured area to employ biofunctional substitutes in which cells are harmonizing with scaffolding and growth factors to create an engineered cartilage tissue.

This chapter reviews the state-of the-art in cartilage tissue engineering focused on tissue engineering approaches designed to recapitulate the native development of cartilage and its tridimensional structure as an osteochondral unit. Since the production of hypertrophied tissue is one of the most critical challenges to overcome in chondral tissue regeneration, here we show new strategies to minimize hypertrophy in cartilage. Finally, the efficacy and safety of different treatments of cartilage in current clinical trials will be discussed.

While the framework provides new features and benefits concerning the strategies for articular tissue regeneration, this chapter presents a set of tools to improve approaches to orthopedic regenerative medicine based on the use of MSCs.
\end{abstract}

Keywords: MSCs, MSCs-subpopulations, cartilage regeneration, cartilage tissue engineering, hypertrophy 


\section{Introduction}

The chondrocytes are the only cells found in cartilage. The chondrocytes demonstrate distinctive properties such as being metabolically active in order to maintain the renewal of the extracellular matrix (ECM) by synthesizing collagens, proteoglycans, hyaluronic acid, and glycoproteins. Restoration of the cartilage damage is still challenging for orthopedic medicine due to its poor ability to regenerate [1].

Mesenchymal stem cells (MSCs) have potential applications in tissue engineering, and regenerative medicine represents an attractive option for repairing lesions in cartilage. Stem cellbased therapies that harmonize with tissue-engineering technologies, and biomaterials are vital for the continuous advance of cartilage regenerative medicine [2, 3].

Once the relationship between structure function in normal and damaged tissues is understood and the development of biological substitutes for the repair or regeneration can be reached. To develop a biological substitute, tissue engineering uses scaffolds, cells, and growth factors. Each of these elements alone is able to promote tissue regeneration, but composites fabricated in combination would be more effective $[4,5]$.

The objective of the present chapter is, therefore, to describe the cellular and molecular framework in which chondrocyte differentiation develops and the articular tissue responds to the injury.

The maintenance of the chondrogenic phenotype during in vitro expansion and avoidance of hypertrophy of MSC-derived chondrocytes remains a challenge in cell-based strategies. Since chondrocyte differentiation is regulated by various signalling pathways, including fibroblast growth factor (FGF), transforming growth factor- $\beta$ (TGF- $\beta$ ), and wingless/Int (WNT)/ $\beta$-catenin, the role of these growth factors is analyzed. Furthermore, we show some strategies to minimize hypertrophy in cartilage [6-8].

Even though an ideal protocol for cartilage regeneration is yet to be established, approaches involving cells, biomaterials, and technology of tissue engineering will advance firmly toward effective clinical application.

\section{Cartilage tissue}

Cartilage is a type of connective tissue whose function is to protect the bones of the diarthrodial joints from the frictional forces associated with the load and impact support [1]. Hyaline or articular cartilage is heterologous, with varying density and organization according to the depth of its zones [9]. Articular cartilage is predominantly avascular, aneural, and alymphatic, so the main route for nutrition is through synovial fluid and assisted by mechanical compression forces [10].

The articular cartilage forms a thin layer of tissue with variable thickness depending on the body location. In humans, it ranges from 1 to $4 \mathrm{~mm}$ depending on the joint [11]. This tissue has viscoelastic ability, giving it the characteristic of deforming in order to increase the total contact 
surface with the consequent reduction of stress and increase the resistance to damage caused by applied loads. This function depends on the organization of the macromolecules in the extracellular matrix, particularly the arrangement and orientation of the collagen fibers [12].

Articular cartilage possesses a coefficient of friction between 0.002 and 0.02 ; the quality of the synovial fluid, the elastic deformation of the cartilage, and the effusion of the liquid from it are the factors able to decrease it. There are also factors that increase the coefficient of friction, such as alteration in the continuity at the surface of the cartilage (fibrillation) [13].

\subsection{Chondrocytes}

Cartilage consists of a single type of specialized cells called chondrocytes, representing approximately $5-10 \%$ of the tissue [14]; chondrocytes are embedded and clustered in the extracellular matrix (ECM) secreted by themselves (Figure 1A). Its function is influenced by changes in the ECM itself, as much as by changes in cell membrane pressure, age, and certain growth factors. These cells depolymerize and eliminate the ECM to enlarge their lacunae, a process featured in the endochondral ossification [13]. They are also present in isolation or organized in isogenic groups, depending on their metabolic activity, i.e., the cells that have just divided are active chondrocytes possessing a very large Golgi apparatus and a basophilic cytoplasm, which means that it can be stained with basic stains with net positive charge such as hematoxylin. These characteristics indicate that a protein synthesis is being performed and are initially located in the same lacunae, but as they secrete new intercellular matrix, they are separated; on the contrary, initial chondrocytes with low or no activity have a clear cytoplasm and a small Golgi apparatus [13].

\subsection{Extracellular matrix}

More than $98 \%$ of the articular cartilage corresponds to the ECM. The extracellular matrix is a dynamic network of macromolecules self-assembled. It is composed of water, gases, metabolites, cations and predominantly of collagens, noncollagenous glycoproteins, hyaluronan, and proteoglycans. ECM is able to regulate cell behavior influencing its proliferation and maturation processes. Therefore, it is not only scaffolding for chondrocytes but also functioning as a reservoir for growth factors and cytokines and modulates the cell activation state (Figure 1A) [13].

\subsubsection{Water}

The water occupies between 60 and $80 \%$ of the ECM volume; its function is to allow the deformation of the cartilage in response to stress, and it is also important for cartilage nutrition and joint lubrication. Approximately $30 \%$ of the water is contained in the intrafibrillar space within the collagen; however, a small percentage fills the intracellular space. The rest is concentrated in the pore space of the matrix. Interestingly, the ability of the articular cartilage to withstand significant loads comes from the frictional resistance to water flow and the pressurization of water within the matrix. When the amount of water increases up to $90 \%$, as in the osteoarthritis (OA), it causes increased permeability, which in turn causes a decrease in resistance and compromises elastic abilities [13]. 
A
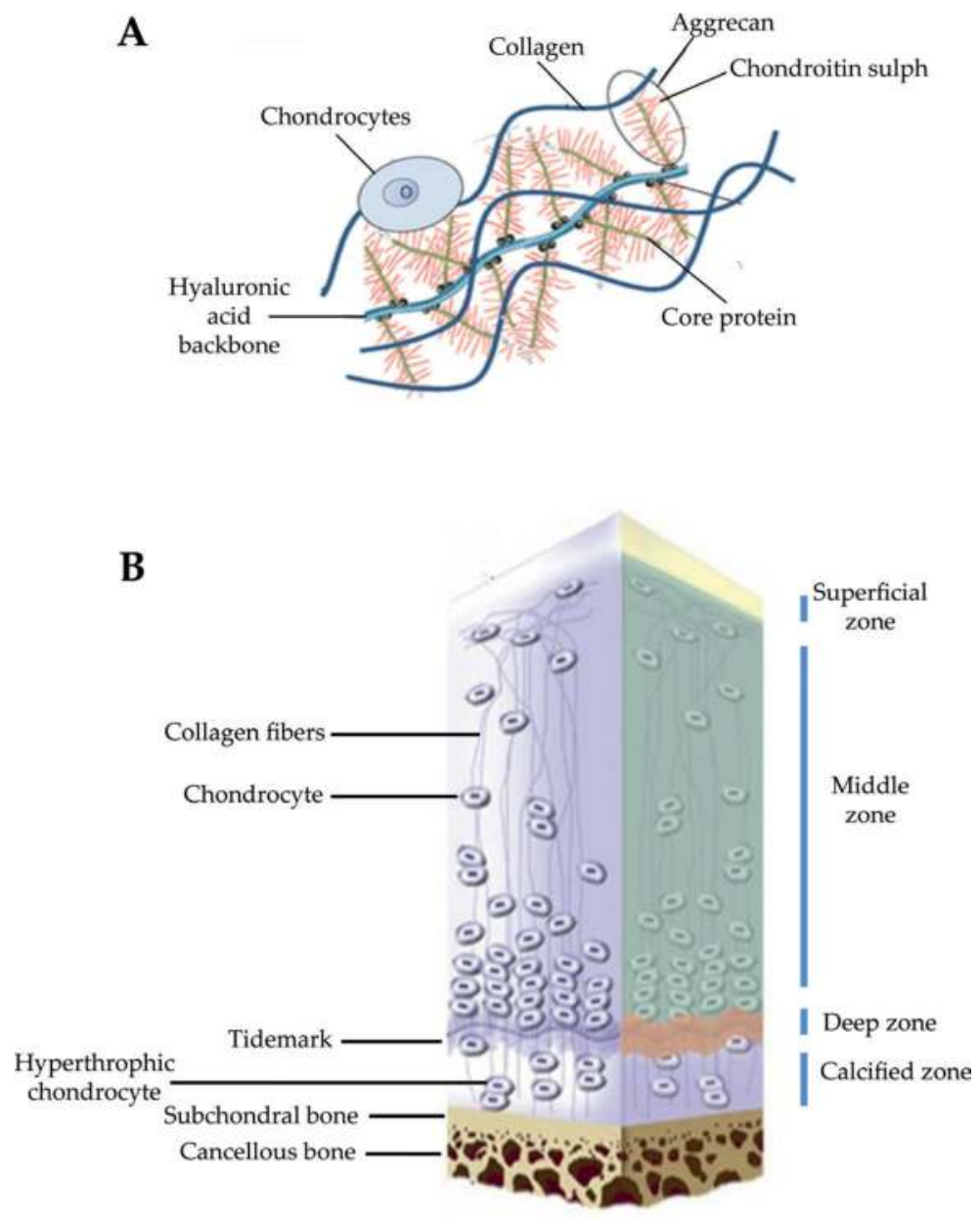

Figure 1. Components of ECM and 3D structure of the articular cartilage. (A) Model of extracellular matrix proteins showing their interaction with each other: collagens (mostly type II collagen); proteoglycan monomers, GAGs as aggrecan, and chondroitin sulphate covalently bound to the core protein. The proteoglycan monomers are assembled onto hyaluronic acid to form aggregates of proteoglycans. (B) The 3D organization of normal articular cartilage: the four areas of the cartilage are highlighted. The superficial zone, where flattened chondrocytes are located; the middle zone containing elongated chondrocytes; the deep zone, where the chondrocytes are arranged in columns and at the bottom, the calcified zone. (A) is modified from Izadifar et al. [15], and (B) from Minas et al. [16].

\subsubsection{Collagen}

Collagen is the most abundant structural macromolecule in ECM, and it makes up about $60 \%$ of the dry weight of cartilage. Collagen types present in cartilage are I, II, IV, V, VI, IX, and XI; however, type II collagen represents $90-95 \%$ of the total amount. Type II collagen forms fibrils, and fibers intertwines with proteoglycan, while the minor collagens stabilize the fibril 
network. Collagen $X$ alone is present in osteochondral ossification phases and is therefore associated with cartilage calcification. Collagen VI is thought to form hexagonal nets in the vicinity of cells where it can bind to collagen II fibrils through matrilin-4, and biglycan nevertheless can be found in OA processes [17].

\subsubsection{Proteoglycans}

Proteoglycans (PGs) represent $10-15 \%$ of the ECM and are the main noncollagenous proteins present in cartilage. These macromolecules secreted by chondrocytes are responsible for the compression of cartilage. PGs are composed of one or more linear glycosaminoglycan (GAGs) chains covalently attached. The most frequent GAG is chondroitin sulfate (of which there are two subtypes, chondroitin-4-sulfate and chondroitin-6-sulfate), then keratan sulfate (or keratan sulfate or keratin sulfate), and dermatan sulfate. GAGs bind to a protein core, thus structuring the aggrecan. These aggrecans are bound by hyaluronic acid-binding proteins (a nonsulfated GAG) to form aggregates of PGs. It is important to mention that chondroitin4-sulfate decreases over the years, whereas chondroitin-6-sulfate remains constant over the years, on the other hand, keratan sulfate and dermatan sulfate tend to increase with age. The half-life of PGs is 3 months, having the ability to retain water being responsible for the porous structure of cartilage [17].

Aggrecan occupies the interfibrillar space of the cartilage ECM and gives cartilage its osmotic properties, a critical feature to its ability to resist compressive loads [17].

The nonaggregating proteoglycans decorin, biglycan, and fibromodulin are also able to interact with collagen. These molecules differ from glycosaminoglycan in composition and function. Decorin and fibromodulin interact with the type II collagen fibrils and have a role in fibrillogenesis and interfibril interactions, whereas biglycan mainly interacts with collagen VI (Figure 1A) [18].

\subsection{Extracellular glycoproteins}

Among these are anchorine CII, fibronectin, laminin, and integrins. Their functions are to connect to the chondrocytes with the ECM, whereas integrins are the most important since they are able to interact with cellular receptors and influence migration, proliferation, and differentiation of chondrocytes [19].

Cartilage usually has protease inhibitors that help in the continuous renewal of the ECM constituent. The composition of ECM varies depending on the cartilage layer and the proximity to the chondrocytes, in this way, PGs rich in keratan-sulfate accumulate in the internal ECM, whereas in the territorial ECM, PGs rich in chondroitin sulfate are abundantly found.

Integrins into focal adhesions contribute to the activation of signaling pathways in the cell, promoting changes in cell survival, proliferation, and gene expression [20]. In vitro studies demonstrate that chondrocytes may interact with various proteins of the ECM such as fibronectin, laminin, vitronectin, osteopontin, bone sialoprotein II, and collagen types I, II, and VI through different integrins [21, 22]. 


\subsection{Tridimensional structure of the articular cartilage}

Till this point, we have reviewed the cellular and molecular components of the articular tissue, but how are they connected to each other?

The articular cartilage has a complex three-dimensional structure. Chondrocytes, collagen fibers, and ECM allow separate articular cartilage into various zones: the superficial zone, the middle zone, the deep zone, and the calcified zone (Figure 1B). The space between these zones allows in turn identifying three regions: the pericellular region, the territorial region, and the interterritorial region.

The superficial zone is thin and protects deeper layers from shear stresses. Mainly composed of types II and IX collagen packed tightly and aligned parallel to the articular surface (Figure 1B), contains flattened chondrocytes, which are in contact with synovial fluid. This zone is responsible for the tensile properties of cartilage. Below the superficial zone, the middle (transitional) zone is found, representing a bridge between the superficial and deep zones. The middle zone contains spherical chondrocytes at low density, proteoglycans, and thicker collagen fibrils. The middle zone is responsible for resistance to compressive forces. The deep zone provides the highest resistance to compressive forces. In the deep zone are found the largest diameter collagen fibrils in a radial arrangement and a low quantity of water. The chondrocytes are organized in columnar orientation, parallel to the collagen fibers and perpendicular to the joint line. Finally, the calcified layer of hypertrophic chondrocytes attaches the cartilage to the bones through anchoring the collagen fibrils of the deep zone to subchondral bone. The tidemark discriminate the deep zone from the calcified cartilage.

\section{Cartilage injuries and osteoarthritis}

Articular cartilage injuries are able to stimulate significant musculoskeletal morbidity in young and in aging patient populations. Restoration of joint damage to date represents a major challenge for medicine since they cannot heal spontaneously, and over time can also lead to the development of osteoarthritis.

The grading of articular cartilage lesions is performed through instrumented palpation of the lesion and via direct observation by arthroscopy [23,24]. The most complete grading system is established by the International Cartilage Repair Society (ICRS) [25]. The ICRS grading system is based on the depth of the lesion and the degree to which subchondral bone is involved and classified the lesion as follows: Grade 0 for a normal joint; Grade 1 for nearly normal, featured by superficial lesions, soft indentation, and/or superficial fissures and cracks; Grade 2 for abnormal lesions extending down to $<50 \%$ of cartilage depth; Grade 3 for severely abnormal lesions where cartilage defects are extending down to $>50 \%$ of cartilage depth as well as down to calcified layer and down to but not through the subchondral bone; and Grade 4 for severely abnormal lesions where blisters are included [25].

Articular cartilage has limited ability for intrinsic repair. The injured chondrocytes (by a superficial or partial-thickness injuries) since early stages have a defective metabolic capacity and 
unable to maintain the normal PG concentration. This triggers other modifications in the tissue, such as increased tissue hydration and fibrillar disorganization of the collagen [26, 27]. These changes favor an increased transmission force to the subchondral bone. Exceeding the capacity of the subchondral bone makes the impact on the damaged cartilage even greater. In response to the events, chondrocytes proliferate, and thus production of matrix molecules at the site of injury increases; however, the new matrix fails to restore the native surface [26]. When the injury reaches subchondral bone (full-thickness injuries), the inflow of pluripotent marrow elements is observed [28]. The migrating mesenchymal stem cells produce type I collagen fibers to fill the full-thickness defect with fibrocartilage. Fibrocartilage fails to provide the necessary functions needed by the articular cartilage [29].

The strategies for articular cartilage lesions treatment can be classified into palliative such as physiotherapy and systemic pain relief medications; reparative such as debridement, knee joint lavage, arthroscopic abrasion arthroplasty, microfracture, and marrow stimulation techniques; restorative such as high tibial osteotomy, unicompartmental knee arthroplasty, and total knee arthroplasty; and transplantation such as osteochondral transplantation (osteochondral grafting), mosaicplasty, and autologous chondrocyte transplantation [27, 30].

Osteoarthritis $(\mathrm{OA})$ is a chronic joint disorder classified into primary and secondary according to the etiology. OA is characterized by the progressive breakdown of the articular cartilage likewise changes in the subchondral bone, synovium, and muscles [31]. In early-stage OA, remodeling and bone loss of both trabecular and cortical subchondral areas are enhanced, while late-stage $\mathrm{OA}$ is featured by remodeling and an increased subchondral plate densification [32].

Mesenchymal stem cells (MSCs) are a promising option for the treatment of OA. MSCs are multipotent progenitor cells with self-renewal abilities, high plasticity, and anti-inflammatory properties; moreover, the capability to differentiate into different lineages including chondrocytes [33].

Despite extensive preclinical research with promising results, because of its technical limitations such as definition on the optimal cell source, processing, assembly with scaffolding, and administration modality, the use in patients is not yet overwhelming, and the design of a systematic procedure is still to be addressed [34, 35, 153, 154].

\section{Cartilage tissue engineering}

The application of cells into scaffolds, as tissue engineering do, makes cartilage regeneration strategies complex but allows to orchestrate the process efficiently [155]. Tissue engineering (TI) can be defined as the combined use of cells, biomaterials, and chemical factors to repair injured or diseased tissues. At the moment, it combines the contribution of cells that are placed on a scaffold, where the factors that accelerate its proliferation can be added; this composite is then transplanted at the site of the lesion in order to achieve tissue regeneration [36].

TI has the potential to provide long-lasting solutions to tissue damage and tissue loss, and engineering cartilage is not an exception to this approach. In fact, due to its limited ability to self-repair, cartilage is an ideal candidate for tissue engineering. 
The critical point of the strategies based on TI is the expansion of cells in culture to be able to generate more cells for the production of tissue in vitro or for the implantation of cells in suspension or on scaffolds for regeneration of the tissue in vivo [37]. Another challenge to overcome regarding this strategy is to achieve the merging of the composite after implantation. The integration of the implanted tissue with the organ requires remodeling, degradation, and formation of new tissue. The remodeling of the implanted tissue is essential for its functionality [37].

TI makes possible the in vitro tissue synthesis, and then, the functional abilities of the composite can be evaluated before implantation. The main disadvantage is the partial absence of physiological and mechanical stimuli during their formation, which does not allow an adequate cellular regulation and spatial development of the tissue, and the decrease of its mechanical quality is observed as a consequence.

In the past decades, the strategies were designed without considering the cartilage as a complex tissue with a functionally that stratified three-dimensional structure. Nowadays, efforts are focused on achieving the landmarks in the process of cartilage formation with the development of a multiphase implant that recapitulates the cartilage as an osteochondral unit [156].

Cartilage tissue engineering combines a cell source, biomaterials, and growth or differentiation factors. Useful cell sources include autologous chondrocytes, minced autologous cartilage, and mesenchymal stem cells (from bone marrow, muscle, synovium, or adipose) $[4,6,7$, 24-28]. Regarding scaffolds, they may be fabricated with natural (e.g., collagen) or synthetic materials and designed as monophasic (chondral phase) or multiphasic (imitating the osteochondral unit) [6-8, 23, 38-41, 157, 158].

Chondroinductive growth factors are essentially members of the transforming growth factor- $\beta$ (TGF- $\beta$ ) superfamily, some members of fibroblast growth factor (FGF) family and insulin-like growth factor-1 (IGF-1). These growth factors can be added to a culture medium to induce chondrogenic differentiation or through gene delivery, and, more recently, by nanoparticle delivery [4, 27, 29-32].

\subsection{Cell source}

Cell-based therapy is a biological therapy, involving the use of cells to develop new tissues or repair damage tissues. Therapies have been designed in order to generate a neocartilage in an attempt to offer the patients with chondral injuries an improvement in the quality of life or a long-lasting cure.

Autologous implantation of chondrocytes (ACT), intra-articular injection of meniscus with stem cells, and autologous matrix-induced chondrogenesis represent the current strategies for cartilage repairing by cell-based therapies. Furthermore, approaches using cell therapy with tissue engineering and biomaterials are increasing [42].

The optimal cell source for cartilage tissue engineering is not yet well established. The goal is to select a cell source that can be isolated by simple methods, are able to expand, and capable of being cultured to synthesize cartilage-specific molecules. The sources range from chondrocytes, fibroblasts, and stem cells to genetically modified cells [159-161]. 
Although chondrocytes have been the most used at first since they are found in native cartilage and have been extensively studied, they currently do not represent the best option.

Likewise, fibroblasts are easily and abundantly obtained and under treatment with lactic acid, they can acquire a chondrogenic phenotype.

Furthermore, stem cells can be expanded through several passages maintaining the differentiation potential. Additionally, all of these cells can be modified genetically to induce or enhance chondrogenesis. Adult MSCs are able to differentiate into osteoblasts, adipocytes, muscles, and chondrocytes and are, therefore, a suitable cellular source for tissue regeneration. Recent evidence indicates that there is great variability in the ability of differentiation between stem cells from specific tissues.

\subsubsection{Chondrocytes}

To perform autologous chondrocytes implantation, a full-thickness sample from a low-weightbearing region of the joint is taken by biopsy during a first arthroscopic operation to collect a chondrocyte population.

The recovered tissue is enzymatically treated to isolate chondrocytes, which are then ex vivo expanded under conditions that preserve cell viability till yielding 12-48 million cells [43].

During a second operation, the chondrocytes are implanted into the debrided cartilage defect. This technique avoids potential immune complications or viral infections from transplanting allogeneic cells or foreign materials [44]. Nevertheless, two operations are needed, and a long recovery time (6-12 months) is required to ensure neotissue maturation.

Several studies have shown that chondrocytes "dedifferentiate" into fibrochondrocytes in culture [45]. However, according to Martinez et al., they can redifferentiate and express chondrocytic markers after being cultured into a 3D in vitro culture system [46].

Moreover, ex vivo culturing of the chondrocytes reduced production of type II collagen and PGs upon expansion in monolayer culturing [47]. This process has been known as dedifferentiation, so the analysis of different markers of chondrocytic maturity as BMP-2, FGFR-3, and COL2A1 is necessary to confirm a stable chondrocyte phenotype.

The growth and the expression of type II collagen have been assessed after autologous chondrocytes implantation to substantiate the expansion of chondrocytes. These markers were found ineffective in predicting the capacity of expanded cells to produce stable cartilage tissue [48].

Xenogeneic and allogeneic chondrocyte have been studied as alternative chondrocytes cell sources. However, these cells can be involved in the induction of immune responses and diseases transmission. Thus, more studies are needed to overcome such issues in the field of allogeneic and xenogeneic chondrocytes.

\subsubsection{Mesenchymal stem cells}

MSCs have higher proliferation rates than chondrocytes and possess a vast differentiation potential toward a chondrogenic, also they are easy to collect from several tissues, such as 
adipose tissue, synovial membrane, and bone marrow. In addition, MSCs produces several extracellular matrix macromolecules involved in cartilage function, including fibronectin, collagen(s), PGs, and glycosylaminoglycans (GAGs), as well as a wide range of cytokines, growth factors, chemokines, and colony stimulating factors [49].

\subsubsection{Bone marrow-derived MSCs}

Bone marrow-derived stem cells (BM-MSCs) are one of the relevant stem cell choices for tissue engineering, and different studies have reported a potential of these cells for cartilage repair and as a treatment of the osteoarthritis. BM-MSCs can be differentiated into chondrocytes in a variety of culture conditions, usually involving induction with TGF- $\beta$ and in a tridimensional environment (e.g., cell pellets and micromasses). The addition of TGF- $\beta$ enhances chondrogenesis; however, the degree of chondrogenesis depends on the culture method or scaffolding [50]. In addition to TGF- $\beta$, other growth factors as BMP-6 and IGF- 1 during in vitro culture also affect chondrogenesis as evidenced by enhanced type II collagen and aggrecan expression and accumulation [51].

Coculture system with chondrocytes is another approach used to promote chondrocyte differentiation of MSCs. Cell proliferation and positive expression of type II collagen have been observed, and this is due to growth factor secretions and cell-cell interactions as well as the microenvironment created by the chondrocytes [52].

Limitation on the use of BM-MSCs is the mechanical integrity of the matrix they produce which is poor in GAGs content. Moreover, in BM-MSCs undergoing chondrogenic induction, a high expression of COLX, MMP13, and ALP markers was observed by in vitro pellet culture; this profile frames a hypertrophic process. Likewise, stem cells derived from adipose tissue (AD-MSCs) were also associated with the development of hypertrophy, as demonstrated by type $X$ collagen over-expression and up-regulation of ALP activity [53].

\subsubsection{Adipose tissue-derived MSCs}

Adipose tissue-derived MSCs (AD-MSCs) have a mesenchymal lineage as demonstrated by the expression of surface markers, such as CD105, CD73, and CD90, and posses the potential to differentiate into chondrocytes. Differentiation is achieved under a high density as micromass cultures or embedded in scaffolds in the presence of TGF- $\beta$, ascorbate, and dexamethasone, especially in combination with a tridimensional culture environment [54].

Lai et al. analyze chondrogenic potential of human adipose-derived stem cells (hAD-MSCs) using three-dimension biomimetic hydrogels. In addition, the effect of TGF- $\beta 3$ supplementation was also included. They demonstrated that in the presence of TGF- $\beta 3$, the expression levels of aggrecan and type II collagen expression were significantly up-regulated. However, expression levels of type I- and X-collagen were also significantly enhanced, which indicates a fibrotic repair [55].

To overcome the fibrotic repair, Zhu et al. developed a strategy of programmed application of TGF 33 and NSC23766 (a Rac1 inhibitor) to commit the hyaline cartilage differentiation of adipose-derived stem cells (AD-MSCs) for joint cartilage repair. The efficacy of AD-MSCs 
with programmed application of TGF $\beta 3$ and Rac1 inhibitor for cartilage regeneration was analyzed in a rat model of osteochondral defects. The results showed that TGF $\beta 3$ promoted AD-MSCs chondro-lineage differentiation, and that the administration of NSC23766 after 7 days postindcution prevented AD-MSC-derived chondrocytes from hypertrophy in vitro and in vivo (Figure 2) [56].

\section{Protein} Markers

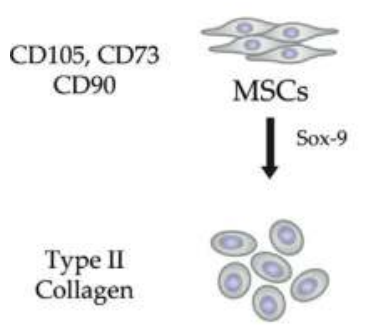

Proliferating chondrocyte

MSCs

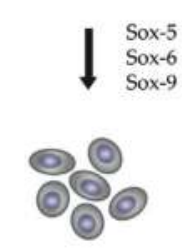

Chondroblast

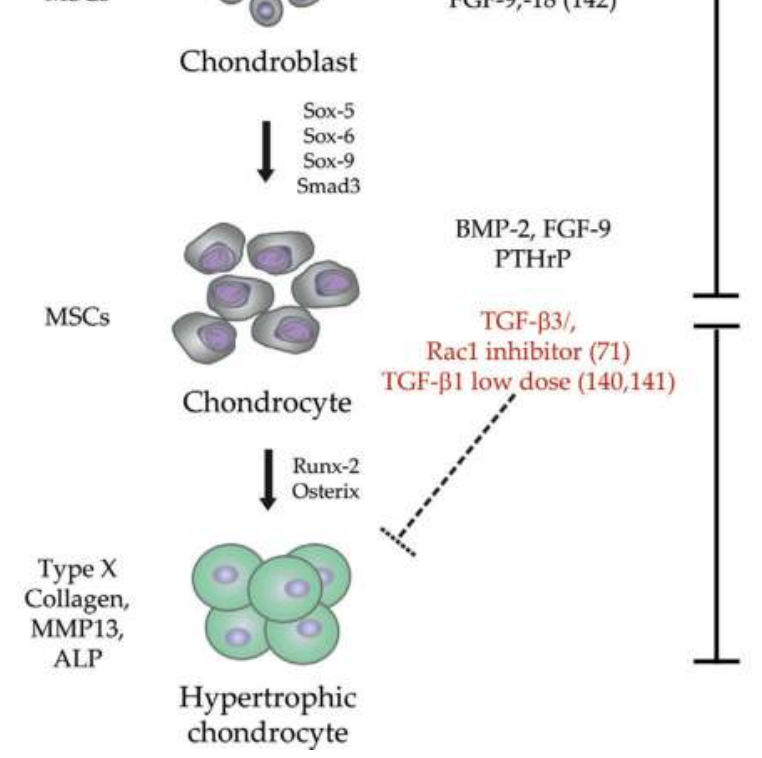

\section{ECM proteins}

TGF- $\beta 1$,

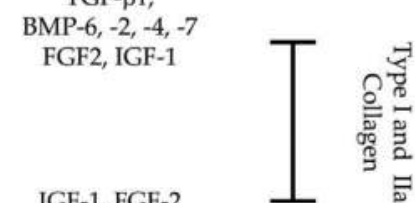

BMP-2,-6, -9 FGF-9,-18 (142) 
In vitro culture, using growth factors like FGF-2 and BMP-6, influences the chondrogenesis of $\mathrm{AD}-\mathrm{MSC}$ as they are able to generate matrix proteins with accumulation of sulfated-GAGs. The use of FGF-2 increases cell proliferation and chondrogenesis through up-regulation of FGF-R2 and Sox 9 [57]. Besides, BMP-6 enhances expression of aggrecan and type II collagen (Figure 2) [58].

\subsubsection{Synovium-derived MSCs}

In the pursuit for a healthy cartilage regeneration through autologous transplantation, it has been discovered that synovial can be a valuable source of SCs for the effective induction of chondrogenesis and the production of high-quality cartilage in vitro [59,60] and in vivo [61].

Synovium-derived MSCs (SDSCs) have a more effective chondrogenic potential than stem cells from other sources. Sakaguchi et al. revealed that human SDSCs have greater expansion and chondrogenic ability in vitro than MSCs from classical sources as bone marrow, periosteum, and adipose tissue. They also note that SDSCs generated larger pellets and a more structured ECM consistent with toluidine blue cartilage matrix staining, concluding that these are a superior source for chondrogenesis than AD-MSCs [62].

Extracellular matrix deposited by SDSCs overcomes two of the main problems related to the development and maturation of chondrocytes: dedifferentiation and chondrocyte redifferentiation [63]. Both processes are beaten because the tissue engineered cartilage matrix secreted by SDSCs is rich in collagen-II and aggrecan but not collagen-I or collagen- $X$ and is mechanically similar to articular cartilage [64].

MSCs culture in general possesses inherent cell heterogeneity; however, for tissue engineering applications, it is imperative to start with a well-defined cell population, particularly, since it has been demonstrated that MSCs subpopulations are featured by a distinct regeneration potential. In this regard, the isolation of subpopulations from SDSCs cultures has been reported using various surface markers in order to sort by flow cytometry.

Arufe et al. reported the isolation from SDSCs of a CD271 $1^{+}$subpopulation which showed high expression of SOX9, aggrecan, and COL2A1 at day 46 of chondrogenic induction; however, the expression of COL10A1 was observed $[35,65]$. Meanwhile, the CD105+ subpopulation reached a homogeneous cellular culture, and it was shown that after a chondrogenic induction, the increase in SOX9 expression was efficiently accompanied by an extracellular matrix rich in type II collagen with no evidence of fibrocartilage $[35,66]$.

More recently, in 2013, another subpopulation with efficient chondrogenic potential was reported, and the $\mathrm{CD} 73^{+} \mathrm{CD} 39^{+}$cell subpopulation showed higher expression levels of SOX9 and a significantly greater chondrogenic potency than the CD73+CD39- cell subpopulation and the original SDSCs population $[35,67]$.

\subsection{Growth and transcription factors in chondrocyte}

Chondrocytes differentiation from MSCs, chondrocyte morphology maintenance, and cartilage matrix formation are processes driven by differentiation and growth factors. A number of extracellular signalling molecules and growth factors as members of the fibroblast growth 
factor (FGF), hedgehog, transforming growth factor- $\beta$ (TGF- $\beta$ ) and bone morphogenic protein (BMP), insulin-like growth factor (IGF), and wingless/Int (Wnt) glycoproteins are all key regulators of chondrocyte cell condensation and chondrogenic differentiation.

Cartilage formation begins by the condensation and then the differentiation of MSCs to prechondrocytes; thus, the cells first express types I and IIA collagen, and begins to synthesize GAGs and adhesion-related proteins such as cadherin [68]. This cascade of events is in response to the effect of factors, such as some members of the TGF- $\beta$ superfamily (TGF- $\beta 1,-\beta 2$, and $-\beta 3$ ), which are able to induce the synthesis of fibronectin, tenascin, and syndecan [69].

As prechondrocytes, the expression of transcription factors as SOX9, L-SOX5, and SOX6 became relevant until prechondrocytes reach the maturation stage and produce an ECM rich in collagen fibers (collagen types II, IX and XI) and PGs [70]. The main indicator of chondrocytes maturation is represented by type II collagen [71].

\subsubsection{Insulin-like growth factor}

Insulin-like growth factor (IGF) is a protein capable of regulating cell growth, differentiation, migration, and survival, and there are two types: IGF-1 and IGF-2. The activity of IGF-1 and IGF-2 (ligands) is collectively regulated by IGF-1R and IGF-2R (receptors), IGF-binding proteases, and IGF-binding proteins.

IGF-1 plays an important role in cell proliferation; it is mainly expressed in the liver although also in brain, heart, lung, bone, placenta, and testes and also produced by chondrocytes [72] thus having an autocrine and/or paracrine regulation [73].

IGFs in the early stages of chondrogenic differentiation induce the proliferation of chondrocytes and stimulate the differentiation of MSCs into prechondrocytes. IGFs act through the type I receptor tyrosine kinase (IGF-1R) that triggers mitogenic activity, regulated by extracellular kinase-kinase signals (MEK, ERK, and MAPK) and via the phosphatidylinositol 3-kinase/Akt (PI3K/Akt) pathway. Several investigations in animal models in vivo demonstrated that IGF significantly promotes the growth and matrix synthesis in articular cartilage and also improves the synthesis of proteoglycans and type II collagen [74].

\subsubsection{The transforming growth factor- $\beta$}

The transforming growth factor- $\beta$ (TGF- $\beta$ ) is a superfamily of polypeptides and contains different factors, including TGF- $\beta$, inhibins, activins, and bone morphogenetic proteins (BMPs); the most promising for cartilage tissue engineering is TGF- $\beta$ and bone morphogenetic proteins (BMPs), highlighting BMP-2, GDF-5, and BMP-7 [75].

TGF- $\beta$ generally induces differentiation of MSCs into chondrocytes, stimulates their proliferation, increases ECM production, and inhibits endochondral ossification.

The three isotypes of TGF- $\beta(\beta 1, \beta 2$, and $\beta 3)$ are secreted in their inactive form and are activated only when dissociated from a peptide associated with latency (LAP). TGF- $\beta$ initiates signaling by binding to the serine/threonine kinase types I and II receptors on the cell surface, 
which then propagates the signal through the phosphorylation of the R-Smad protein (Smad 2 and 3). TGF- $\beta$ signaling is also negatively regulated by I-Smad (Smad 6 and 7), which interferes with R-Smad phosphorylation. TGF- $\beta$ also activates mitogen-activated protein kinases (MAPK) [74].

BMPs are also multifunctional polypeptides having a key role in chondrogenesis by promoting terminal differentiation [76]. During first stages of chondrogenesis, the BMPs induce the expression of the N-cadherin thus promoting cell-cell interaction [77] indispensable for SOX expression. BMP signalling pathway enhances type $\mathrm{X}$ collagen promoter activity resulting in chondrocyte hypertrophy [78]. Furthermore, in vitro culturing BMP promotes the up-regulation of type II collagen and aggrecan [79].

\subsubsection{Fibroblast growth factors}

Fibroblast growth factors (FGF) belong to a family of polypeptides that are involved in several functions including cell proliferation, differentiation, survival, and motility, essential during cartilage growth, development, and repair. FGFs play a crucial role in the maintenance of stem cells and their activation [80]. FGF binding to fibroblast growth factor receptor (FGFR) leads to the activation of signaling pathways, including PI3K, Src, phospholipase C $\gamma$, MAPKs, ERK, and p38. In particular, two members of the FGF family, basic fibroblast growth factor (bFGF, also known as FGF-2) and fibroblast growth factor-18 (FGF-18), play an important regulatory role in maintaining homeostasis of the cartilage matrix [74].

\subsubsection{Vascular endothelial growth factor}

Vascular endothelial growth factor (VEGF) is a hormone that seems to be important in the cartilage growth plate, where it supports the formation of endochondral bone, apparently by attracting endothelial cells from the bone marrow. It has been speculated that this factor could promote the mitotic cycle of chondrocytes, although little is known about this feature [81].

\subsubsection{Platelet-derived growth factor}

Platelet-derived growth factor (PDGF) is a potent mitogenic and chemotactic factor for all cells of mesenchymal origin, including chondrocytes and mesenchymal stem cells. It is related to increased cell proliferation and proteoglycan production [82].

\subsubsection{Transcription factors}

Many transcription factors are involved during chondrogenesis and endochondral ossification depending on the location of the chondrocytes within the articular cartilage. There are three main transcription factors involved in chondrogenesis: Sox9, L-Sox5, and Sox6.

Sox9 is involved in the condensation phase of MSCs into prechondrocytes when it is expressed in cells that initiate an aggregation that afterward stimulates the expression of cartilagespecific markers and inhibits terminal differentiation of chondrocytes. Sox 9 also induces the expression of the L-SOX5 and SOX6 transcription factors, which definitely compromise the MSCs to develop in the chondrogenic lineage [83]. 


\subsection{Scaffolds}

\subsubsection{Biomaterials}

A significant research has been focused toward developing biomaterials that can mimic cartilage matrix, thus restoring function at the defect site. The biomaterials should satisfy three significant criteria: mechanical properties according to those of existing cartilage, integration with adjacent cartilage, and adequate biodegradability.

Natural biomaterials have the advantage as better cellular interaction due to the presence of ligands that can facilitate adhesion, in addition to promoting the activation of various chondrogenic activation pathways. The most common natural biomaterials are collagen, gelatine, lysozyme, Matrigel ${ }^{\mathrm{TM}}$, hyaluronic acid, fibrin, chitosan, agarose, and alginate [84].

As chondrocytes are surrounded by a hyaluronan-based pericellular matrix, they have been designed as hydrogels consisting of hyaluronan [85]. Chondrocytes attach firmly to hyaluronan-based matrices, stimulating as a result the matrix production [86]. Despite these advantages, the newly formed matrix is characterized by insufficient mechanical integrity [87].

Alginate is a natural biopolymer derived from brown algae and is composed of homopolymeric blocks of L-glucuronic acid and D-manuronic acid [88] and is widely used in biomedicine due to its biocompatibility and low toxicity [89]. Such materials require the use of divalent gelling cations to form a network structure, which allows the cells used to promote tissue regeneration to be trapped [90].

In contrast to natural polymers, synthetic polymers provide a better control of the structural and mechanical features. Polyglycolic acid (PGA) and polylactic acid (PLA) are the most used since they degrade by hydrolysis at rates depending on the selecting monomers [91]. A major disadvantage of synthetic polymers is that they do not offer specific biological functions [92]. Its functionalization with biological motifs or bioactive molecules facilitates cell adhesion and consequently stimulating matrix production [76].

Fragments derived from the cartilage have been also used for application in joint regeneration; however, it is mandatory to eliminate the cellular component to avoid an immune response when implanted in an in vivo model; therefore, they are decellularized by different methods [93, 94]. It has been observed that they support the production of type II collagen and proteoglycans; in addition, they minimize the hypertrophy of the newly formed tissues with the cooperation of growth factors [95].

\subsubsection{Biological scaffolds and its use in the treatment of chondral lesions}

A better understanding of the molecular structure and functional role of extracellular matrix components in the physiology of the cartilage [96] supported the construction of scaffolds which mimick cartilage microenvironment.

Scaffold-based approaches possess several advantages compared to scaffold-free: such as increased control to fill the cartilage defect according to the features and size of the lesion; no surgical procedures are required to obtain tissue from the patient; increased graft stability that influence recovery time for the patient. Most important, since the chondrocytes are 
cultured in a 3D environment, dedifferentiation is a minimal concern, thus, the cells are able to produce a more hyaline-like cartilage [97].

The geometry and microarchitecture of scaffolds are important factors that determine cell adhesion and migration, as well as the preservation of the synthesized matrix. [98]. In addition, the pore size is a critical feature; it should be large enough to allow the migration of the cells and thus promote the production of ECM, [99] but small enough to establish a large surface area for cell adhesion. It is recommended to generate scaffolds with a pore size ranging between 300 and $350 \mathrm{~nm}$ [100].

Successful cartilage regeneration is closely related to the ability of the scaffold to support the chondrocyte proliferation rate and to the differentiation of MSCs within a tissue-engineered 3D matrix [101]. At the same time, it becomes imperative to characterize the quality of the expanded MSC as well as to avert the development of hypertrophic chondrocytes [49, 102].

\section{Minimizing the development of hypertrophic tissue in cell-based therapies}

Chondrogenic hypertrophy is characterized by an increase of the cell volume as well as remodeling of ECM [103]. The increase in the volume is the result of intracellular and extracellular osmolarity variations where aggrecans are the main contributors.

The hypertrophic differentiation of chondrocytes is a process that gradually leads to the mineralization of cartilage. The main factors involved are the transcription factor runt-related transcription factor 2 (RUNX2) and myocyte enhancer factor-2C (MEF2C). Both promote the expression of proteins that determine terminal differentiation, including matrix metalloproteinase 13 (MMP13) [104], type X collagen [105], alkaline phosphatase (ALP), and vascular endothelial growth factor (VEGF) [106]; all of them functionally contribute to endochondral ossification. MMP13 is a key modulator in this process as it degrades type II collagen and aggrecan [107]. Furthermore, type $X$ collagen serves as a framework for the calcification through matrix vesicles [108], ALP hydrolyzes pyrophosphate to inorganic phosphate that, in the presence of calcium, forms hydroxyapatite (Figure 2) [109].

Among the growth factors that are directly involved in the process are transforming growth factors (TGFs) and bone morphogenetic proteins, which are able to initiate cartilage differentiation but often lead to hypertrophy and calcification, since TGF $\beta 3$ is also the active signal pathway during endochondral ossification [110].

Moreover, Woods et al. has reported that Rac1 functions as a positive regulator in governing chondrocyte hypertrophy, maturation, and calcification [111] through up-regulation of type $X$ collagen, MMP13, and ADTAMTS-5 that induce hypertrophy and chondrocyte calcification [112].

Therefore, Rac1 is an important target for controlling the development of hypertrophy has been demonstrated that the inhibition of Rac1 activity overcomes not only chondrocyte hypertrophy and calcification but also alleviates osteoarthritis progression [56, 112-115]. 
TGF- $\beta$ s and BMPs bind to specific receptors to recruit type I receptor to trigger signalling of their specific receptor-Smads. The importance of Smad-dependent TGF- $\beta$ - and BMP-signalling pathways for cartilage and bone formation has been well established whose synergic or antagonistic function depends on the microenvironment [116].

However, the control of the activity of some members of the TGF superfamily as TGF- $\beta 1$ is important to control the subsequent maturation of chondrocytes and the consequent mineralization [117]. TGF $\beta 1$ is a key factor in the maintenance of chondrocyte phenotype. BMP9 and TGF $\beta 1$ dose-dependently synergized on Smad2 phosphorylation and showed an additive effect on expression of Smad3-dependent genes. Furthermore, the addition of a low dose of TGF $31(1 \mathrm{ng} / \mathrm{ml})$ diminishes expression of early markers of cellular hypertrophy Alpl and type $X$ collagen (Figure 2) [118, 119].

Likewise, in vitro addition of TGF $\beta 3$ in the later stages of differentiation has been shown to control the fibrotic process. Pei et al. found that in synovium-derived stem cells (SDSCs), TGF- $\beta 3$ enhancing collagen II and sulfated glycosaminoglycan minimize the expression of collagen I in the repair of partial-thickness cartilage defects in porcine SDSC pellets compared with TGF- $\beta 1$ [120].

Meanwhile, blocking of BMP signalling during chondrogenesis of MSC restricts Type X collagen and MMP13 expression from cartilage at maintained collagen type II and enhanced SOX9 expression [116]. Thus, the manipulation of BMP-signalling (essentially BMP4) is able to shift chondrogenesis of the MSCs toward a nonhypertrophic phenotype. Dexheimer et al. addressed this concern by the inhibition of Smad1/5/9-signalling using dorsomorphin [121]. This seems to be a good strategy to potentiate chondrogenesis and also inhibit hypertrophic differentiation; however, now the efforts should be focused on establishing a timeline on specific cellular models at which point the inhibition of $\mathrm{pSmad1/5/9}$ signaling should be carried out. Inhibition of pSmad1/5/9 signalling apparently stopped chondrogenesis or decelerated MSC differentiation toward hypertrophy depending on the time of treatment initiation.

FGF factors play an important regulatory role in maintaining homeostasis of the cartilage matrix [74, 164]. Correa et al. designed an elegant sequential protocol based on the addition of FGF2, 9, and 18 on bone marrow-derived hMSC. The growth factors are added as follows: increased cell proliferation and priming (FGF2 [d0 to d3]); stimulated early chondrogenic differentiation by shifting the chondrogenic program earlier (TGF- $\beta$, FGF9/FGF18 [d4 to d14]); enhanced ECM production (d14 to d21); and delayed terminal hypertrophy (FGF9/FGF18 [d21 to d28]). To highlight, in the proterminally differentiating conditions, both FGF9 and FGF18 were able to reduce Runx2 expression and the activity of the hypertrophy-specific marker alkaline phosphatase (ALP) (Figure 2) [122].

As has already been established, TGF- $\beta$ and bone morphogenetic protein are key factors for chondrogenesis. They are capable of initiating signals in mitogen-activated protein kinase (MAPK) pathway essentially of extracellular signal-regulated kinase ERK-1 and ERK-2 [123, 124].

PD98059 is one of the ERK inhibitors that suppressed hypertrophy in the chondrogenesis from bone marrow-derived hMSCs, inhibiting the cascade signalling upstream of ERK1/2 activation [125, 126]. Lee et al. [123] constructed a PD98059-impregnated poly(lactic-co-glycolic acid) 
(PLGA) scaffold and demonstrated that it effectively suppresses the hypertrophy of hBMMSCs that have been differentiated toward the chondrocytic lineage in basic chondrogenic medium (Dulbecco's modified Eagle's medium/Ham's F-12 nutrient mixture: DMEM/F-12 supplemented with $1 \%$ insulin-transferrin-sodium selenite [ITS], $50 \mu \mathrm{M}$ ascorbate-2-phosphate, $1 \times 10^{-7} \mathrm{M}$ dexamethasone, $1 \mathrm{mM}$ sodium pyruvate, and $50 \mu \mathrm{M}$ l-proline). The efficiency of the system was challenged in a rabbit model where the main observations were the abundant presence of type-II collagen in ECM with the absence of types I and type X collagens [123].

As described in the above example, scaffolds can additionally be a useful tool for the release of elements that both regulate the differentiation process and control the development of fibrotic tissue. Inhibitors are not the only elements that can be incorporated into scaffolding systems; the addition of growth factors to scaffold systems has also been reported to reduce the development of hypertrophic chondrocytes, with successful results.

Mimicking the native tissue architecture is critical for effective cartilage regeneration. Kim et al. developed a multifunctional system based on TGF- $\beta 3$ encapsulated PLCL scaffold using human adipose-derived stem cells (ADSCs) to promote chondrogenesis. They demonstrated the release of TGF- $\beta 3$ molecules for 8 weeks, which remained in the PLCL matrix. Furthermore, this scaffolding system formed a hyaline cartilage-specific lacunae structure and minimized the hypertrophy of differentiated chondrocytes [127].

Fibrocartilage is a dense, fibrous version of a cartilage. It has been shown that a lack of integration of the graft in the lesion area promotes poor functional properties and limits complete regeneration of the defect.

The scaffold design should offer hierarchical structure, desired mechanical properties (stiffness, elasticity, permeability, and diffusion), and ability for adapting into the anatomical shapes. The use of scaffolds that are composed of one type of biomaterial, with homogeneous porosity and architecture, and used a single-cell type limits the integration with the surrounding tissue and encourages a fibrotic process. Instead, the design of stratified or gradient scaffold mimics the structural and mechanical features of a native osteochondral unit. In order to achieve stratification and composition, composite scaffolds are assembled through a multilayered scaffold design; in this way, structural templates for the cartilaginous layer, the tidemark, and calcified cartilage, and the subchondral bone are generated [163].

A biphasic scaffold design was reported, based on a silk-protein scaffold constituting the cartilage phase and a silk-coated strontium-hardystonite-gahnite ceramic scaffold constituting the bone phase, and both phases are cellularized with human mesenchymal stem cells (hMSCs). For the biphasic scaffold, there were noticeable to significant increases in Sox-9, collagen type II, and aggrecan in addition to low type X collagen expression levels compared with the chondral single-phase version of the implant [39].

Ho et al. designed a biphasic implant comprising of a polycaprolactone (PCL) cartilage scaffold and a PCL-tricalcium phosphate as bone scaffold; it was seeded with mesenchymal stem cells (MSCs), and the cartilage and bone constructs were maintained in the MSC expansion media prior to implantation into critically sized osteochondral defects in a pig model. After 6 months, the cartilaginous repair was observed with a low occurrence of fibrocartilage. Furthermore, the functional cartilage restoration was demonstrated by a high Young's modulus [40]. 
The chondral phase was derived from bovine decellularized articular cartilage ECM, while the osseous phase was composed of a PLGA/ $\beta$-TCP wrapped with type I collagen. The biphasic scaffolds was cellularized by BMSCs induced with chondrogenic and osteogenic medium and implanted into osteochondral defects in a rabbit knee model. By a histological evaluation, the presence of an uniform neocartilage surface, a clear fusion of neocartilage, a regenerated subchondral bone with a well-defined tidemark, and no evidence of fibrocartilage was demonstrated [41].

\section{The efficacy and safety of different treatments of cartilage in current clinical trials}

Current surgical treatments for symptomatic cartilage lesions include reparative and reconstructive treatments. The former employ techniques that stimulate tissue cells to form hyaline cartilage, such as the microfracture technique, perforation, abrasion arthroplasty, and biological procedures involving cell culture, such as autologous chondrocyte implantation (ACI), of the latter derives the development of biocompatible three-dimensional scaffolds, where chondrocytes, stem cells, or chondrocyte-like cells can be grown. The latter involves mosaicplasty, such as the transplantation of autografts or allografts, which are composed of mature hyaline cartilage attached to the subchondral bone [128]. The type of treatment to be used will depend on the size of the lesion, the location, and the type of activity of the patient.

ACI has been the treatment most often used to treat large knee cartilage injuries. Previous studies have compared ACI with mosaicplasty, microfracture, and matrix-guided microfracture; where the ACI obtains better results (88\%) than mosaicplasty $(69 \%)$, presenting hyaline cartilage in half of the biopsies; however, statistically significant results are restricted to the medial condylar area [129]. ACI has been compared to microfracture and mosaicplasty but has never been compared with simple arthroscopic debridement and rehabilitation alone. At present, the first study that will detail, with a high level of evidence, the results of comparing ACI with simple debridement and physiotherapy in symptomatic lesions of full thickness of the knee is being carried out. This study aims to increase the clinical and economic knowledge between these techniques in the short and long term [130].

Recently, surgical treatments have been complemented with the use of autologous biological materials such as PRP and mesenchymal stem cells [162]. Recently, the use of autologous PRP coupled with the microfracture technique has been associated with better short-term clinical and functional outcomes, especially in pain [131-133]; in addition, arthroscopic implantation of AD-MSC combined with the microfracture technique has also been associated with a decrease in pain [134]. Similarly, the use of AD-MSC [135, 136] or BM-MSC [137, 138] has been reported as a safe therapeutic alternative via intraarticular injection in patients with osteoarthritis, which report a significant improvement in pain levels. Another complementary therapy is the use of hyaluronic acid, which improves articular cartilage repair in combination with autologous peripheral blood stem cells via intraarticular postoperative injection, complementing the technique of arthroscopic subchondral perforation [139]. 
Gene therapy techniques represent an alternative strategy for gene transfer for therapeutic purposes. Through gene therapy, proteins are synthesized at the site of the defect or injury where they directly influence the natural microenvironment. Growth factors most frequently synthesized by engineered cartilage cells to regenerate damaged cartilage include BMPs, IGF-1, and TGF- $\beta$ s 1,2 , and 3.

The application of genetically engineered cartilage in clinical trials begins to generate results. Mont et al. have reported on a series of clinical trials in which the effect of injecting genetically engineered chondrocytes virally transduced with TGF- $\beta 1$ (GEC-TGF- $\beta 1$ ) into the knees of 54 patients with osteoarthritis has been evaluated. After 12 or 24 weeks of treatment, patients reported a degenerative process delayed compared to the placebo. It should be noted that the studies demonstrating cartilage regeneration are missing [140].

Moreover, they also evaluate the efficacy to safely regenerate cartilage by a phase II clinical trial in 102 (ClinicalTrials.gov NCT01221441) [141] and 27 patients (ClinicalTrials.gov NCT02341378) [142], respectively. Patients expressed decreased pain and improvement in function and physical capacity; thus, treatment injection of GEC-TGF- $\beta 1$ seems to improve symptoms and pain due to knee osteoarthritis.

Although gene therapy is very promising for cartilage repair, much remains to be understood about the clinical results obtained. It is crucial to control several aspects such as the duration of transgenic expression, as well as the identification and selection of a therapeutic factor that is clinically useful for cartilage regeneration.

With the boom in tissue engineering in recent years and innovation in the area of biomaterials, doctors have new options for treating chondral lesions. To date, the clinical use of these materials is limited; very few polymers have been used for clinical trials in cartilage tissue engineering. The list includes collagen, polymers based on hyaluronan and fibrin, because they have a biomimetic structure similar to the native articular cartilage. Chondral or osteochondral grafts consist in the surgical transfer of mature tissue to a cartilage defect. The graft could be an autologous tissue transfer from a nonload-bearing zone and cultivated on a porated scaffold.

Within this narrow list is highlighted the collagen, which is the main component of ECM. Zheng et al. have analyzed the efficacy of an implant constructed with chondrocytes cultured onto collagen type I/III scaffolds in 56 patients with OA. The results mainly evidenced the maintenance of a chondrocytic phenotype, as well as a good integration of the implant in the injured area and the production of aggrecan and type II collagen. These data demonstrated the regeneration of hyaline cartilage tissue 6 months after treatment [143]. Furthermore, based on the information contained in the website, clinicaltrials.gov, clinical trials using stem cells cultivated mainly on collagen scaffolds are ongoing.

Assor et al. conduct a clinical trial to demonstrate the efficacy and safety of BM-MSCs cultured on a collagen type I scaffold dotted with hydroxyapatite to regenerate articular cartilage defects of the knee (Table 1 NCT01159899). Otherwise, Giannini et al. studied the critical points of the regenerative treatment with BM-MSCs embedded in equine collagen type I scaffold (Table 1 NCT02005861). Both trials are still in process and have not shown final results. 


\begin{tabular}{|c|c|c|c|c|c|}
\hline $\begin{array}{l}\text { Clinical trials.gov } \\
\text { identifier }\end{array}$ & Phase & Brief description & Status & No. patients & $\begin{array}{l}\text { Principal } \\
\text { investigator }\end{array}$ \\
\hline NCT00850187 & Phase 1 & $\begin{array}{l}\text { Autologous } \\
\text { transplantation } \\
\text { of mesenchymal } \\
\text { stem cells (MSCs) } \\
\text { cultured on a } \\
\text { collagen I scaffold } \\
\text { in full-thickness } \\
\text { articular cartilage }\end{array}$ & Completed & 6 & $\begin{array}{l}\text { Leila } \\
\text { Taghiyar, }\end{array}$ \\
\hline NCT01159899 & Early Phase 1 & $\begin{array}{l}\text { Autologous } \\
\text { BM-MSCs, } \\
\text { stimulated } \\
\text { with a protein } \\
\text { matrix cultured } \\
\text { in a collagen } \\
\text { hydroxyapatite } \\
\text { scaffold }\end{array}$ & Unknown & 50 & Michel Assor \\
\hline NCT02005861 & Not provided & $\begin{array}{l}\text { Bone marrow } \\
\text { aspirate concentrate } \\
\text { cultivated onto an } \\
\text { equine collagen } \\
\text { type } 1 \text { scaffold } \\
\text { (IOR-G1, Novagenit, } \\
\text { Mezzolombardo, } \\
\text { TN, Italy) }\end{array}$ & Recruiting & 140 & $\begin{array}{l}\text { Sandro } \\
\text { Giannini }\end{array}$ \\
\hline NCT02659215 & Not provided & $\begin{array}{l}\text { Bone marrow } \\
\text { aspirate concentrate } \\
\text { mixed with a } \\
\text { hyaluronan-based } \\
\text { scaffold (Hyalofast }{ }^{\circledR} \text { ) }\end{array}$ & $\begin{array}{l}\text { Active, not } \\
\text { recruiting }\end{array}$ & 200 & Alberto Gobbi \\
\hline NCT01282034 & Phase 4 & $\begin{array}{l}\text { Bioceramic, } \\
\text { multilayered } \\
\text { scaffold: Type I } \\
\text { equine collagen } \\
\text { and Magnesium } \\
\text { enriched- } \\
\text { Hydroxyapatite } \\
\text { (MaioRegenv®) }\end{array}$ & Completed & 145 & $\begin{array}{l}\text { Maurilio } \\
\text { Marcacci }\end{array}$ \\
\hline NCT01471236 & Phase 4 & $\begin{array}{l}\text { A cell-free system } \\
\text { based on a biphasic, } \\
\text { porous resorbable } \\
\text { scaffold. Cartilage } \\
\text { phase consist in a } \\
\text { modified aragonite } \\
\text { and HA, while bone } \\
\text { phase in aragonite } \\
\text { (Agili-C implant). }\end{array}$ & Recruiting & 65 & Elizaveta Kon \\
\hline
\end{tabular}

Source: https://clinicaltrials.gov.

Table 1. Current clinical trials based on cartilage tissue engineered approaches for the treatment of chondral and osteochondral diseases. 
Hyaluronate is also reported in the clinical trials; Gobbi leads a study to evaluate the efficiency of Hyalofast ${ }^{\circledR}$, together with bone marrow aspirate containing the MSCs without the need for processes for cell isolation and ex vivo expansion. This methodology has the disadvantage that the number of MSCs destined for the repair of the chondral tissue is not controlled nor the cellularity of the aspirate (Table 1 NCT02659215).

New studies evaluate the performance, stability, safety, and viability of the surgical procedure using biomimetic osteochondral implants. One of the most studied and relevant procedure that is currently marketed as MaioRegen ${ }^{\circledR}$ (Table 1 NCT01471236); this is a three-phase implant that considers the biomechanics of the chondral phase, the tidemark, and subchondral bone. Its composition is based on a collagen type-I hydroxyapatite gradient. MaioRegen ${ }^{\circledR}$ has demonstrated throughout its clinical studies that its technique is safe and that the clinical results in the short- and medium-term follow-up are effective in a large population of patients [144-148], even attributed better results than other commercial scaffolds ChondroMimetic ${ }^{\mathrm{TM}}$ [149] and TruFit $\mathrm{CB}^{\mathrm{TM}}[11,150-152]$. Despite the encouraging in vitro results of a wide variety of osteochondral scaffolds, most are in the early stages of development.

In general, clinical trials present current techniques for treating chondral lesions as safe. However, in order to determine the best surgical option in the treatment of symptomatic chondral defects, a rigorous clinical trial should be developed, where there is prospective control, randomization control, even feeding control, and rehabilitation control so that on the basis of comparative results, the surgeon provides an effective treatment.

\section{Conclusion}

This chapter presents an overview of the advances in the design of new cell-based therapies in conjunction with the tools that the tissue engineering offers, such as the use of biomaterials, the selection of subpopulations, and the addition in a temporal manner of growth factors.

It is important to emphasize that the understanding of the molecular mechanisms that governs the chondrocyte differentiation allows generating strategies that reflect a balance between the chondrocyte maturation and the containment in the development of fibrotic repair tissue.

Indeed, the therapeutic approaches for the repair and regeneration of joint tissue should consider as a goal mimic the osteochondral structure, which will result in an effective and safe clinical application.

\section{Acknowledgements}

The authors are supported by a grant from CONACyT; 233365-SALUD/2014. 


\section{Abbreviations}

ACI

AD-MSCs

ALP

ACT

BM-MSCs

BMP

ECM

ERK1/2

FGF

FGFR

GAGs

ICRS

IGF

LAP

MMP13

MSCs

MAPK

MEF2C

$\mathrm{OA}$

PDGF

PRP

PCL

PGA

PLA

PLCL

PLGA

PGs

RUNX2

SDSCS

TI

TGF- $\beta$

VEGF autologous chondrocyte implantation

adipose tissue-derived MSCs

alkaline phosphatase

autologous implantation of chondrocytes

bone marrow-derived stem cells

bone morphogenic protein

extracellular matrix

extracellular signal-regulated kinase -1 and -2

fibroblast growth factor

fibroblast growth factor receptor

glycosaminoglycan

International Cartilage Repair Society

insulin-like growth factor

latency-associated peptide

matrix metalloproteinase 13

mesenchymal stem cells

mitogen-activated protein kinases

myocyte enhancer factor-2C

osteoarthritis

platelet-derived growth factor

platelet-rich plasma

polycaprolactone

polyglycolic acid

polylactic acid

poly(L-lactide-co-caprolactone)

poly(lactic-co-glycolic acid

proteoglycans

Runt-related transcription factor 2

synovium-derived MSCs

tissue engineering

transforming growth factor- $\beta$

vascular endothelial growth factor 


\section{Author details}

Lizeth Fuentes-Mera $^{1 *}$, Alberto Camacho ${ }^{1,2}$, Nidia K. Moncada-Saucedo ${ }^{1}$ and Víctor Peña-Martínez ${ }^{3}$

*Address all correspondence to: lizeth46@hotmail.com

1 Departamento de Bioquímica y Medicina Molecular, Universidad Autónoma de Nuevo León (UANL), Monterrey, México

2 Unidad de Neurociencias, Centro de Investigación y Desarrollo en Ciencias de la Salud, Universidad Autónoma de Nuevo León (UANL), Monterrey, México

3 Servicio de Ortopedia y Traumatología, Universidad Autónoma de Nuevo León (UANL), Monterrey, NL, México

\section{References}

[1] Longo UG, et al. Stem cells and gene therapy for cartilage repair. Stem Cells International. 2012;2012:1-9

[2] Chen CW, et al. Type I and II collagen regulation of chondrogenic differentiation by mesenchymal progenitor cells. Journal of Orthopaedic Research. 2005;23:446-453

[3] van der Kraan PM, et al, Interaction of chondrocytes, extracellular matrix and growth factors: Relevance for articular cartilage tissue engineering. Osteoarthritis and Cartilage. 2002;10:631-637

[4] Chen $\mathrm{WH}$, et al. Tissue engineered cartilage using human articular chondrocytes immortalized by HPV-16 E6 and E7 genes. Journal of Biomedical Materials Research. Part A. 2006;76:512-520

[5] Verbruggen G, et al. Osteochondral repair in synovial joints. Current Opinion in Rheumatology. 2007;19:265-271

[6] Hunziker EB, et al. Articular cartilage repair: Basic science and clinical progress. A review of the current status and prospects. Osteoarthritis and Cartilage. 2002;10:432-463

[7] Kuo CK, et al. Cartilage tissue engineering: Its potential and uses. Current Opinion in Rheumatology. 2006;18:64-73

[8] Heng BC, et al. Directing stem cell differentiation into the chondrogenic lineage in vitro. Stem Cells. 2004;22:1152-1167

[9] Jadin KD, et al. Depth-varying density and organization of chondrocytes in immature and mature bovine articular cartilage assessed by 3D imaging and analysis. The Journal of Histochemistry and Cytochemistry. 2005;53:1109-1119

[10] Knight M, et al. Development of articular cartilage. Osteoarthritis and Cartilage. 2013;21:S2-S3 
[11] Matsiko A, et al. Advanced strategies for articular cartilage defect repair. Materials (Basel). 2013;6:637-668

[12] Eyre D. Collagen of articular cartilage. Arthritis Research. 2002;4:30-35

[13] Sopena J, et al Estructura y función del cartílago articular. Portada: Armas Frente a la Patología Articular. 2000;1:24-26

[14] Chung C, et al. Engineering cartilage tissue. Advanced Drug Delivery Reviews. 2008; 60:243-262

[15] Izadifar Z, et al. Strategic design and fabrication of engineered scaffolds for articular cartilage repair. Journal of Functional Biomaterials. 2012;3(4):799-838

[16] Minas T. Chapter 4: Patient evaluation, cartilage defect, and evidence putting it all together. Musculoskeletal Key

[17] Fox S, et al. The basic science of articular cartilage: Structure, composition, and function. Sports Health. 2009;1(6):461-468

[18] Roughley PJ. The structure and function of cartilage proteoglycans. European Cells \& Materials. 2006;12:92-101

[19] Loeser RF. Integrins and chondrocyte-matrix interactions in articular cartilage. Matrix Biology. 2014;39:11-16

[20] Brodkin KR, et al. Chondrocyte phenotypes on different extracellular matrix monolayers. Biomaterials. 2004;25:5929-5938

[21] Dürr J, et al. Localization of beta 1-integrins in human cartilage and their role in chondrocyte adhesion to collagen and fibronectin. Experimental Cell Research. 1993;207: 235-244

[22] Enomoto $\mathrm{M}$, et al. Beta 1 integrins mediate chondrocyte interaction with type I collagen, type II collagen, and fibronectin. Experimental Cell Research. 1993;205:276-285

[23] Bauer M, et al. Chondral lesions of the femoral condyles: A system of arthroscopic classification. Arthroscopy. 1988;4:97-102

[24] Peterson L, et al. Two- to 9-year outcome after autologous chondrocyte transplantation of the knee. Clinical Orthopaedics and Related Research. 2000;374:212-234

[25] Brittberg $M$, et al. Evaluation of cartilage injuries and repair. The Journal of Bone and Joint Surgery. American Volume. 2003;85-A(Suppl 2):58-69

[26] Mankin HJ. The response of articular cartilage to mechanical injury. The Journal of Bone and Joint Surgery. American Volume. 1982;64:460-466

[27] Lewis $\mathrm{PB}$, et al. Basic science and treatment options for articular cartilage injuries. The Journal of Orthopaedic and Sports Physical Therapy. 2006;36(10):717-727

[28] Goldberg VM, et al. Biologic restoration of articular surfaces. Instructional Course Lectures. 1999;48:623-627 
[29] Nehrer S, et al. Histologic analysis of tissue after failed cartilage repair procedures. Clinical Orthopaedics and Related Research. 1999;365:149-162

[30] Karnes J, et al. Cell therapy for the creation of cartilage and related clinical trials. In: Templeton NS, editor. Gene and Cell Therapy: Therapeutic Mechanisms and Strategies. 4th ed. Taylor \& Francis/CRC Press; 2015. pp. 1123-1135

[31] Loeser RF, et al. Osteoarthritis: A disease of the joint as an organ. Arthritis and Rheumatism. 2012;64(6):1697-1707

[32] Burr DB, et al. Bone remodelling in osteoarthritis. Nature Reviews. Rheumatology. 2012;8(11):665-673

[33] Manferdini C, et al. Adipose-derived mesenchymal stem cells exert antiinflammatory effects on chondrocytes and synoviocytes from osteoarthritis patients through prostaglandin E2. Arthritis and Rheumatism. 2013;65(5):1271-1281

[34] Filardo G, et al. Mesenchymal stem cells for the treatment of cartilage lesions: From preclinical findings to clinical application in orthopaedics. Knee Surgery, Sports Traumatology, Arthroscopy. 2013;21(8):1717-1729

[35] Pérez-Silos V, et al. Mesenchymal stem cells subpopulations: Application for orthopedic regenerative medicine. Stem Cells International. 2016;2016:3187491

[36] Langer RS, et al. Tissue engineering: The challenges ahead. Scientific American. 1999;280: 86-89

[37] Spector M. Biomaterials-based tissue engineering and regenerative medicine solutions to musculoskeletal problems. Swiss Medical Weekly. 2008;136:293-301

[38] Marquass B, et al. A novel MSC-seeded triphasic construct for the repair of osteochondral defects. Journal of Orthopaedic Research. 2010;12:1586-1599

[39] Li JJ, et al. A biphasic scaffold based on silk and bioactive ceramic with stratified properties for osteochondral tissue regeneration. Journal of Materials Chemistry. B, Materials for Biology and Medicine. 2015;3(26):5361-5376

[40] Ho ST, et al. The evaluation of a biphasic osteochondral implant coupled with an electrospun membrane in a large animal model. Tissue Engineering. Part A. 2010;16(4):1123-1141

[41] Da H, et al. The impact of compact layer in biphasic scaffold on osteochondral tissue engineering. PLoS One. 2013;8(1):e54838

[42] Shen W, et al. Intra-articular injection of human meniscus stem/progenitor cells promotes meniscus regeneration and ameliorates osteoarthritis through stromal cell-derived factor-1/CXCR4-mediated homing. Stem Cells Translational Medicine. 2014;3(3):387-394

[43] Richardson JB, et al. Repair of human articular cartilage after implantation of autologous chondrocytes. Journal of Bone and Joint Surgery (British). 1999;81:1064-1068

[44] Saris DB, et al. Treatment of symptomatic cartilage defects of the knee: Characterized chondrocyte implantation results in better clinical outcome at 36 months in a randomized trial 
compared to microfracture. The American Journal of Sports Medicine. 2009;37(Suppl. 1): 10S-19S

[45] Darling EM,.et al. Rapid phenotypic changes in passaged articular chondrocyte subpopulations. Journal of Orthopaedic Research. 2005;23:425-432

[46] Martinez I, et al. Redifferentiation of in vitro expanded adult articular chondrocytes by combining the hanging-drop cultivation method with hypoxic environment. Cell Transplantation. 2008;17:987-996

[47] Benya PD, et al. Dedifferentiated chondrocytes re-express the differentiated collagen phenotype when cultured in agarose gels. Cell. 1982;30(1):215-224

[48] Brittberg M, et al. Treatment of deep cartilage defects in the knee with autologous chondrocyte transplantation. The New England Journal of Medicine. 1994;331(14):889-895

[49] Somoza RA, et al. Chondrogenic differentiation of mesenchymal stem cells: Challenges and unfulfilled expectations. Tissue Engineering. Part B, Reviews. 2014;20:596-608

[50] Coleman RM, et al. Hydrogel effects on bone marrow stromal cell response to chondrogenic growth factors. Biomaterials. 2007;28:2077-2086

[51] Indrawattana $\mathrm{N}$, et al. Growth factor combination for chondrogenic induction from human mesenchymal stem cell. Biochemical and Biophysical Research Communications. 2004;320:914-919

[52] Zhou GD, et al. In vivo chondrogenesis of BMSCs at non-chondrogenesis site by cotransplantation of BMSCs and chondrocytes with pluronic as biomaterial. Advanced Biomaterials VI. 2005;288-289:3-6

[53] Hennig T, et al. Reduced chondrogenic potential of adipose tissue derived stromal cells correlates with an altered TGFbeta receptor and BMP profile and is overcome by BMP-6. Journal of Cellular Physiology. 2007;211(3):682-691

[54] Zuk PA, et al. Human adipose tissue is a source of multipotent stem cells. Molecular Biology of the Cell. 2002;13:4279-4295

[55] Lai JH, et al. Interaction between osteoarthritic chondrocytes and adipose-derived stem cells is dependent on cell distribution in three-dimension and transforming growth factor- $\beta 3$ induction. Tissue Engineering. Part A. 2015;21(5-6):992-1002

[56] Zhu S, et al. Programmed application of transforming growth factor $\beta 3$ and Rac1 inhibitor NSC23766 committed hyaline cartilage differentiation of adipose-derived stem cells for osteochondral defect repair. Stem Cells Translational Medicine. 2014;3(10): 1242-1251

[57] Chiou M, et al. Mitogenic and chondrogenic effects of fibroblast growth factor-2 in adiposederived mesenchymal cells. Biochemical and Biophysical Research Communications. 2006;343:644-652 
[58] Estes BT, et al. Potent induction of chondrocytic differentiation of human adiposederived adult stem cells by bone morphogenetic protein 6 . Arthritis and Rheumatism. 2006;54:1222-1232

[59] Pei M, et al. Engineering of functional cartilage tissue using stem cells from synovial lining: A preliminary study. Clinical Orthopaedics and Related Research. 2008;466(8):1880-1889

[60] Jones BA, et al. Synovium-derived stem cells: A tissue-specific stem cell for cartilage engineering and regeneration. Tissue Engineering. Part B, Reviews. 2012;18(4):301-311

[61] Pei M, et al. Repair of full-thickness femoral condyle cartilage defects using allogeneic synovial cell-engineered tissue constructs. Osteoarthritis and Cartilage. 2009;17(6):714-722

[62] Sakaguchi Y, et al. Comparison of human stem cells derived from various mesenchymal tissues: Superiority of synovium as a cell source. Arthritis and Rheumatism. 2005;52(8):2521-2529

[63] Pei M, et al. Extracellular matrix deposited by synovium-derived stem cells delays replicative senescent chondrocyte dedifferentiation and enhances redifferentiation. Journal of Cellular Physiology. 2012;227:2163-2174

[64] Zhou C, et al. Gene expression profiles reveal that chondrogenic progenitor cells and synovial cells are closely related. Journal of Orthopaedic Research. 2014;32(8):981-988

[65] Arufe MC, et al. Chondrogenic potential of subpopulations of cells expressing mesenchymal stem cell markers derived from human synovial membranes. Journal of Cellular Biochemistry. 2010;111(4):834-845

[66] Arufe MC, et al. Differentiation of synovial CD-105+ human mesenchymal stem cells into chondrocyte-like cells through spheroid formation. Journal of Cellular Biochemistry. 2009;108(1):145-155

[67] Gullo F, et al. Prospective purification of a subpopulation of human synovial mesenchymal stem cells with enhanced chondro-osteogenic potency. Rheumatology. 2013;52(10): 1758-1768

[68] Burdan F, et al. Morphology and physiology of the epiphyseal growth plate. Folia Histochemica et Cytobiologica. 2009;47:5-16

[69] Chimal J, et al. Expression of N-cadherin, N-CAM, fibronectin and tenascin is stimulated by TGF-beta1, beta2, beta3 and beta5 during the formation of precartilage condensations. International Journal of Developmental Biology. 1999;43:59-67

[70] Goldring MB, et al. The control of chondrogenesis. Journal of Cellular Biochemistry. 2006;97:33-44

[71] Demoor M, et al. Cartilage tissue engineering: Molecular control of chondrocyte differentiation for proper cartilage matrix reconstruction. Biochimica et Biophysica Acta. 2014;1840:2414-2440

[72] Gaissmaier C, et al. Growth and differentiation factors for cartilage healing and repair. Injury. 2008;39:S88-96 
[73] Chen $Y$, et al. Insulin-like growth factor-1 boosts the developing process of condylar hyperplasia by stimulating chondrocytes proliferation. Osteoarthritis and Cartilage. 2012;20:279-287

[74] Shanmugarajan T, et al. Growth factors and signaling pathways in the chondrogenic differentiation of mesenchymal stem cells. Tissue Engineering \& Regenerative Medicine. 2011;8:292-299

[75] Fortier L, et al. The role of growth factors in cartilage repair. Clinical Orthopaedics and Related Research. 2011;469:2706-2715

[76] Vinatier C, et al. Cartilage engineering: A crucial combination of cells, biomaterials and biofactors. Trends in Biotechnology. 2009;27(5):307-314

[77] Haas AR, et al. Chondrogenic differentiation of murine C3H10T1/2 multipotential mesenchymal cells: II. Stimulation by bone morphogenetic protein-2 requires modulation of N-cadherin expression and function. Differentiation. 1999;64(2):77-89

[78] Fernández-Lloris R, et al. Induction of the Sry-related factor SOX6 contributes to bone morphogenetic protein-2-induced chondroblastic differentiation of C3H10T1/2 cells. Molecular Endocrinology. 2003;17(7):1332-1343

[79] Gründer T, et al. Bone morphogenetic protein (BMP)-2 enhances the expression of type II collagen and aggrecan in chondrocytes embedded in alginate beads. Osteoarthritis and Cartilage. 2004;12(7):559-567

[80] Shi S, et al. Growth factor transgenes interactively regulate articular chondrocytes. Journal of Cellular Biochemistry. 2013;114:908-919

[81] Aurich M, et al. Histological and cell biological characterization of dissected cartilage fragments in human osteochondritis dissecans of the femoral condyle. Archives of Orthopaedic and Trauma Surgery. 2006;126:606-614

[82] Zapata N, et al. Cultivo de tejido cartilaginoso articular: Acercamiento conceptual. Environmental Impact Assessment Review. 2007;8:117-129

[83] Akiyama $\mathrm{H}$, et al. The transcription factor Sox9 has essential roles in successive steps of the chondrocyte differentiation pathway and is required for expression of Sox 5 and Sox6. Genes \& Development. 2002;16:2813-2828

[84] Glowacki J et al. Collagen scaffolds for tissue engineering. Biopolymers. 2008;89: 338-344

[85] Guilak F, et al. The pericellular matrix as a transducer of biomechanical and biochemical signals in articular cartilage. Ann N Y Acad Sci. 2006;1068:498-512

[86] Foss C, et al. Silk fibroin/hyaluronic acid 3D matrices for cartilage tissue engineering. Biomacromolecules. 2013;14(1):38-47

[87] Nagaya H, et al. Examination of synovial fluid and serum hyaluronidase activity as a joint marker in rheumatoid arthritis and osteoarthritis patients (by zymography). Annals of the Rheumatic Diseases. 1999;58(3):186-188 
[88] Hori Y, et al. Modular injectable matrices based on alginate solution/microsphere mixtures that gel in situ and co-deliver immunomodulatory factors. Acta Biomaterialia. 2011;5:969-982

[89] Park H, et al. Facile control of RGD-alginate/hyaluronate hydrogel formation for cartilage regeneration. Carbohydrate Polymers. 2011;86:1107-1112

[90] Bhat A, et al. Alginate hydrogels containing cell-interactive beads for bone formation. FASEB Journal. 2013;27:4844-4852

[91] Stoop R. Smart biomaterials for tissue engineering of cartilage. Injury. 2008;39:S77-87

[92] Shen Y, et al. Biomaterial and mesenchymal stem cell for articular cartilage reconstruction. Current Stem Cell Research \& Therapy. 2014;9(3):254-267

[93] Tavassoli A, et al. Bovine articular cartilage decellularized matrix as a scaffold for use in cartilage tissue engineering. Iranian journal of Veterinary Science and Technology. 2012;4:1-8

[94] Elder BD, et al. Extraction techniques for the decellularization of tissue engineered articular cartilage constructs. Biomaterials. 2009;30:3749-3756

[95] Diekman B et al. Chondrogenesis of adult stem cells from adipose tissue and bone marrow: Induction by growth factors and cartilage-derived matrix. Tissue Engineering. Part A. 2010;16:27-31

[96] Harvey A, et al. Proteomic analysis of the extracellular matrix produced by mesenchymal stromal cells: implications for cell therapy mechanism. PLoS One. 2013;8:11

[97] Caron MM, et al. Redifferentiation of dedifferentiated human articular chondrocytes: Comparison of 2D and 3D cultures. Osteoarthritis and Cartilage. 2012;20:1170-1178

[98] Oh SH, et al. Investigation of pore size effect on chondrogenic differentiation of adipose stem cells using a pore size gradient scaffold. Biomacromolecules. 2010;127:1948-1955

[99] Chen FH, et al. Technology insight: Adult stem cells in cartilage regeneration and tissue engineering. Nature Clinical Practice. Rheumatology. 2006;2:373-382

[100] O'Brien FJ, et al. The effect of pore size on cell adhesion in collagen-GAG scaffolds. Biomaterials. 2005;26:433-441

[101] Grigolo B, et al. Evidence for redifferentiation of human chondrocytes grown on a hyaluronan-based biomaterial (HYAff 11): Molecular, immunohistochemical and ultrastructural analysis. Biomaterials. 2002;23:1187-1195

[102] Hoch AI, et al. Concise review: Optimizing expansion of bone marrow mesenchymal stem/stromal cells for clinical applications. Stem Cells Translational Medicine. 2014;3:643-652

[103] Bush PG, et al. The osmotic sensitivity of rat growth plate chondrocytes in situ; clarifying the mechanisms of hypertrophy. Journal of Cellular Physiology. 2008;214(3):621-629 
[104] Studer D, et al. Molecular and biophysical mechanisms regulating hypertrophic differentiation in chondrocytes and mesenchymal stem cells. European Cells \& Materials. 2012;24:118-135

[105] Arnold MA, et al. MEF2C transcription factor controls chondrocyte hypertrophy and bone development. Developmental Cell. 2007;12(3):377-389

[106] Mueller MB, et al. Functional characterization of hypertrophy in chondrogenesis of human mesenchymal stem cells. Arthritis and Rheumatism. 2008;58(5):1377-1388

[107] Fosang AJ, et al. Degradation of cartilage aggrecan by collagenase-3 (MMP-13). FEBS Letters. 1996;380(1-2):17-20

[108] Shen G. The role of type X collagen in facilitating and regulating endochondral ossification of articular cartilage. Orthodontics \& Craniofacial Research. 2005;8(1):11-17

[109] Van Donkelaar CC, et al. The PTHrP-Ihh feedback loop in the embryonic growth plate allows PTHrP to control hypertrophy and Ihh to regulate proliferation. Biomechanics and Modeling in Mechanobiology. 2007;6(1-2):55-62

[110] Tamamura Y, et al. Developmental regulation of Wnt/beta-catenin signals is required for growth plate assembly, cartilage integrity, and endochondral ossification. Journal of Biological Chemistry. 2005;280(19):19185-19195

[111] Woods A, et al. Rac1 signaling stimulates N-cadherin expression, mesenchymal condensation, and chondrogenesis. Journal of Biological Chemistry. 2007;282(32):2350023508

[112] Zhu S, et al. Inhibition of Rac1 activity by controlled release of NSC23766 from chitosan microspheres effectively ameliorates osteoarthritis development in vivo. Annals of the Rheumatic Diseases. 2015;74(1):285-293

[113] Xiao J, et al. Regulation of chondrocyte proliferation through GIT1-Rac1-mediated ERK1/2 pathway by PDGF. Cell Biology International. 2014;38(6):695-701

[114] Long DL, et al. Rac1 is required for matrix metalloproteinase 13 production by chondrocytes in response to fibronectin fragments. Arthritis and Rheumatism. 2013;65(6): 1561-1568

[115] Woods A, et al. Rac1 signaling regulates CTGF/CCN2 gene expression via TGFbeta/ Smad signaling in chondrocytes. Osteoarthritis and Cartilage. 2013;17(3):406-413

[116] Hellingman CA, et al. Smad signaling determines chondrogenic differentiation of bone-marrow-derived mesenchymal stem cells: inhibition of Smad1/5/8P prevents terminal differentiation and calcification. Tissue Engineering. Part A. 2011;17: 1157-1167

[117] Shintani N, et al. TGF-ss1 enhances the BMP-2-induced chondrogenesis of bovine synovial explants and arrests downstream differentiation at an early stage of hypertrophy. PLoS One. 2013;8:e53086 
[118] Van Caam A, et al. The high affinity ALK1-ligand BMP9 induces a hypertrophy-like state in chondrocytes that is antagonized by TGF $\beta 1$. Osteoarthritis and Cartilage. 2015;23(6):985-995

[119] Prasadam I, et al. ERK-1/2 and p38 in the regulation of hypertrophic changes of normal articular cartilage chondrocytes induced by osteoarthritic subchondral osteoblasts. Arthritis and Rheumatism. 2010;62:1349-1360

[120] Pei M, et al. Repair of large animal partial-thickness cartilage defects through intraarticular injection of matrix-rejuvenated synovium-derived stem cells. Tissue Engineering. Part A. 2013;19(9-10):1144-1154

[121] Dexheimer V, et al. Differential expression of TGF- $\beta$ superfamily members and role of Smad1/5/9-signalling in chondral versus endochondral chondrocyte differentiation. Scientific Reports. 2016;6:36655

[122] Correa D, et al. Sequential exposure to fibroblast growth factors (FGF) 2, 9 and 18 enhances hMSC chondrogenic differentiation. Osteoarthritis and Cartilage. 2015;23(3):443-453

[123] Lee JM, et al. PD98059-impregnated functional PLGA Scaffold for direct tissue engineering promotes chondrogenesis and prevents hypertrophy from mesenchymal stem cells. Tissue Engineering. Part A. 2014;20(5-6):982-991

[124] Oh CD, et al. Opposing role of mitogen-activated protein kinase subtypes, erk-1/2 and p38, in the regulation of chondrogenesis of mesenchymes. Journal of Biological Chemistry. 2000;25:5613

[125] Kim HJ, et al. The effects of ERK1/2 inhibitor on the chondrogenesis of bone marrow- and adipose tissue-derived multipotent mesenchymal stromal cells. Tissue Engineering. Part A. 2010;16:851

[126] Chen Z, et al. ERK1 and ERK2 regulate chondrocyte terminal differentiation during endochondral bone formation. Journal of Bone and Mineral Research. 2015;30(5):765-774

[127] Kim SH, et al. TGF- 33 encapsulated PLCL scaffold by a supercritical CO2-HFIP co-solvent system for cartilage tissue engineering. Journal of Controlled Release. 2015;206:101-107

[128] Gracitelli GC, et al. Surgical interventions (microfracture, drilling, mosaicplasty, and allograft transplantation) for treating isolated cartilage defects of the knee in adults. Cochrane Database of Systematic Reviews. 2016;9:CD010675

[129] Clar C, et al. Clinical and cost-effectiveness of autologous chondrocyte implantation for cartilage defects in knee joints: Systematic review and economic evaluation. Health Technology Assessment. 2005;9:iii-iv, ix-x, 1-82

[130] Randsborg P-H, et al. Focal cartilage defects in the knee-A randomized controlled trial comparing autologous chondrocyte implantation with arthroscopic debridement. BMC Musculoskeletal Disorders. 2016;17:117 
[131] Mancò A, et al Microfracture versus microfracture and platelet-rich plasma: Arthroscopic treatment of knee chondral lesions. A two-year follow-up study. Joints. 2016;4:142-147

[132] Gormeli G, et al. Clinical effects of platelet-rich plasma and hyaluronic acid as an additional therapy for talar osteochondral lesions treated with microfracture surgery: A prospective randomized clinical trial. Foot \& Ankle International. 2015;36:891-900

[133] Manunta AF, et al. The treatment of chondral lesions of the knee with the microfracture technique and platelet-rich plasma. Joints. 2013;1:167-170

[134] Koh Y-G, et al. Adipose-derived mesenchymal stem cells with microfracture versus microfracture alone: 2-year follow-up of a prospective randomized trial. Arthroscopy: The Journal of Arthroscopic \& Related Surgery. 2016;32:97-109

[135] Pers Y-M, et al. Adipose mesenchymal stromal cell-based therapy for severe osteoarthritis of the knee: A phase I dose-escalation trial. Stem Cells Translational Medicine. 2016;5:847-856

[136] Jo CH, et al. Intra-articular injection of mesenchymal stem cells for the treatment of osteoarthritis of the knee: A proof-of-concept clinical trial. Stem Cells. 2014;32:1254-1266

[137] Vega A, et al. Treatment of knee osteoarthritis with allogeneic bone marrow mesenchymal stem cells: A randomized controlled trial. Transplantation. 2015;99:1681-1690

[138] Orozco L, et al. Treatment of knee osteoarthritis with autologous mesenchymal stem cells. Transplantation. 2013;95:1535-1541

[139] Saw K-Y, et al. Articular cartilage regeneration with autologous peripheral blood stem cells versus hyaluronic acid: A randomized controlled trial. Arthroscopy: The Journal of Arthroscopic \& Related Surgery. 2013;29:684-694

[140] Lee MC, et al. A placebo-controlled randomised trial to assess the effect of TGFB1-expressing chondrocytes in patients with arthritis of the knee. The Bone \& Joint Journal. 2015;97:924-932

[141] Cherian JJ, et al. Preliminary results of a phase II randomized study to determine the efficacy and safety of genetically engineered allogeneic human chondrocytes expressing TGF- $\beta 1$ in patients with grade 3 chronic degenerative joint disease of the knee. Osteoarthritis and Cartilage. 2015;12:2109-2118

[142] Ha CW, et al. A multicenter, single-blind, Phase IIa clinical trial to evaluate the efficacy and safety of a cell-mediated gene therapy in degenerative knee arthritis patients. Human Gene Therapy. Clinical Development. 2015;26(2):125-130

[143] Zheng $\mathrm{MH}$, et al. Matrix-induced autologous chondrocyte implantation (MACI): Biological and histological assessment. Tissue Engineering. 2007;13(4):737-746

[144] Kon E, et al. A novel nano-composite multi-layered biomaterial for treatment of osteochondral lesions: Technique note and an early stability pilot clinical trial. Injury. 2010;41:693-701 
[145] Kon E, et al. Novel nano-composite multilayered biomaterial for osteochondral regeneration: A pilot clinical trial. The American Journal of Sports Medicine. 2011;39:1180-1190

[146] Panseri S, et al. Osteochondral tissue engineering approaches for articular cartilage and subchondral bone regeneration. Knee Surgery, Sports Traumatology, Arthroscopy. 2012;20:1182-1191

[147] Kon E, et al. Clinical results and MRI evolution of a nano-composite multilayered biomaterial for osteochondral regeneration at 5 years. The American Journal of Sports Medicine. 2014;42:158-165

[148] Kon E, et al. A one-step treatment for chondral and osteochondral knee defects: Clinical results of a biomimetic scaffold implantation at 2 years of follow-up. Journal of Materials Science: Materials in Medicine. 2014;25:2437-2444

[149] Getgood AMJ, et al. Evaluation of early-stage osteochondral defect repair using a biphasic scaffold based on a collagen-glycosaminoglycan biopolymer in a caprine model. The Knee. 2012;19:422-430

[150] Dell'Osso G, et al. The biphasic bioresorbable scaffold (Trufit@) in the osteochondral knee lesions: Long-term clinical and MRI assessment in 30 patients. Musculoskeletal Surgery. 2016;100:93-96

[151] Gelber PE, et al. Magnetic resonance evaluation of TruFit ${ }^{\circledR}$ plugs for the treatment of osteochondral lesions of the knee shows the poor characteristics of the repair tissue. The Knee. 2014;21:827-832

[152] Bekkers JEJ, et al. Articular cartilage evaluation after TruFit plug implantation analyzed by delayed gadolinium-enhanced MRI of cartilage (dGEMRIC). The American Journal of Sports Medicine. 2013;41:1290-1295

[153] De Girolamo L, et al. Regenerative approaches for the treatment of early OA. Knee Surgery, Sports Traumatology, Arthroscopy. 2016;24(6):1826-1835

[154] Filardo G, et al. Stem cells in articular cartilage regeneration. Journal of Orthopaedic Surgery and Research. 2016;11:42-56

[155] Caldwell KL, et al. Cell-based articular cartilage repair: The link between development and regeneration. Osteoarthritis and Cartilage. 2015;23(3):351-362

[156] Mollon B, et al. The clinical status of cartilage tissue regeneration in humans. Osteoarthritis and Cartilage. 2013;21:1824-1833

[157] Chiang H, et al. Clinical feasibility of a novel biphasic osteochondral composite for matrix-associated autologous chondrocyte implantation. Osteoarthritis and Cartilage. 2013;21:589-598

[158] Cui L, et al. Repair of articular cartilage defect in non-weight bearing areas using adipose derived stem cells loaded polyglycolic acid mesh. Biomaterials. 2009;30:2683-2693 
[159] Cheng A, et al. Generating cartilage repair from pluripotent stem cells. Tissue Engineering. Part B, Reviews. 2013;20(4):257-266

[160] Diekman BO, et al. Cartilage tissue engineering using differentiated and purified induced pluripotent stem cells. Proceedings of the National Academy of Sciences of the United States of America. 2012;109:19172-19177

[161] Ko JY, et al. In vitro chondrogenesis and in vivo repair of osteochondral defect with human induced pluripotent stem cells. Biomaterials. 2014;35:3571-3581

[162] Mifune Y, et al. The effect of platelet-rich plasma on the regenerative therapy of muscle derived stem cells for articular cartilage repair. Osteoarthritis and Cartilage. 2013;21:175-185

[163] Levingstone TJ, et al. A biomimetic multi-layered collagen-based scaffold for osteochondral repair. Acta Biomaterialia. 2014;10:1996-2004

[164] Barr L, et al. The effect of recombinant human fibroblast growth factor-18 on articular cartilage following single impact load. Journal of Orthopaedic Research. 2014;32: 923-927 
